\title{
Academics can also be culprits of evidence complacency
}

To the Editor - Over the past ten years there has been a growing number of publications attempting to understand the 'gap' between the provision of outputs from academic studies in the fields of applied ecology and conservation science, and their subsequent use by conservation practitioners $^{1}$. William Sutherland has led a team providing global leadership on this difficult issue and in a recent article ${ }^{2}$, together with Claire Wordley, asserted that the science-practitioner gap persists partly because "a culture of evidence complacency remains in many areas of policy and practice". However, we suggest that the charge of evidence complacency should also be levied at academics in this field. Our 'complacency' has perhaps been to assume that our research is of widespread use to practitioners, that is, covers topics that have the ability to inform the efforts of those who are responsible for the day-to-day delivery of conservation actions.

Although we agree with Sutherland and Wordley that "swathes of carefully controlled, peer-reviewed evidence is being generated", its value to practitioners thus far has largely been assumed rather than truly evaluated. And research suggests a problem with the perceived relevance of academic research in many parts of the practitioner sector ${ }^{1}$. This is exacerbated for practitioners by research topics being skewed by pervasive academic cultures, for example, restrictions on applied research funding and the drive for publication impact ratings ${ }^{3}$. So while we agree with the issues identified by Sutherland and Wordley, there is one vital ingredient missing from their suggested solutions. This is the generation of research questions by practitioners (that is, a bottom-up approach) whose outputs would inform decision making processes associated with specific conservation actions.

It should be emphasized that what we are suggesting is different from previous attempts to use large-group processes to identify practice-relevant questions on policy development ${ }^{4}$, global conservation issues ${ }^{5}$ or fundamental ecology ${ }^{6}$. Although they included practitioners, these initiatives have been largely academic-led (top-down). We suggest that removing evidence complacency from both academics and practitioners will require the utility of current scientific outputs to be evaluated. To achieve this, a strategic practitioner-led research agenda will need to be generated.

We recently established an initiative to develop such an agenda with practitioners from over 40 organizations. The project ('What's the point of conservation science') is using a 'competency framework' approach to link the standard range of practitioner tasks (that is, their conservation delivery activities) directly to the very specific evidence required to complete these tasks effectively. To some degree, this approach side-steps the problem of individual opinion and bias often encountered when using group-based (Delphi) techniques ${ }^{7}$. Once developed, this 'research agenda' can then be used as the basis for evaluating the utility of available scientific outputs. This could be in the form of a 'matching exercise' to identify key gaps and priorities, conducted as a partnership between academics and practitioners. Evidence complacency is a feature of many aspects of human endeavour, but with growing pressures on natural systems there has never been a more important time for academics and practitioners to help each other achieve their mutual goals.

\section{Mark O'Connell ${ }^{1 *}$ and Rachel White ${ }^{2}$ ${ }^{1}$ University of Gloucestershire, Swindon Road, Cheltenham, Gloucestershire GL50 4AZ, UK. ${ }^{2}$ University of Brighton, Huxley Building, Lewes Road, Brighton BN2 4GJ, UK. \\ *e-mail:moconnell@glos.ac.uk}

Published online: 2 October 2017

DOI: 10.1038/s41559-017-0346-9

References

1. Knight, A. T. et al. Conserv. Biol. 22, 610-617 (2008).

2. Sutherland, W. J. \& Wordley, C. F. R. Nat. Ecol. Evol. 1, 1215-1216 (2017).

3. Caudron, A., Vigier, L. \& Champigneulle, A. J. Appl. Ecol. 49, 753-757 (2012).

4. Sutherland, W. J. et al. J. Appl. Ecol. 43, 617-627 (2006).

5. Sutherland, W. J. et al. Conserv. Biol. 23, 557-567 (2009).

6. Sutherland, W. J. et al. J. Ecol. 101, 58-67 (2013).

7. Mukherjee, N. et al. Methods Ecol. Evol. 6, 1097-1109 (2015).

Competing interests

The authors declare no competing financial interests. 